\title{
Abdominal Wall Reconstruction Following Strangulated Recurrent Incisional Hernia During Pregnancy
}

\author{
Heath A. Dorion ${ }^{\mathrm{a}, \mathrm{c}}$, Barbara M. Hileman ${ }^{\mathrm{b}}$
}

\begin{abstract}
By the 10-year follow-up, incisional hernias appear at a rate of $18.7 \%$, but it is still unknown how often this complication occurs during pregnancy. It is challenging to diagnose a hernia in a pregnant patient, and there is a lack of literature reporting on these cases. We present the case of a 26 -week gestation, morbidly obese patient who presented with a strangulated, recurrent incisional hernia. She required bowel resection and multiple subsequent incisional hernia repairs. Finally, a bioprosthetic reinforced component separation repair was used on the fifth hernia occurrence. The patient remains free of recurrent hernia but still experiences pain in her abdomen and back. An ideal technique for repairing incisional hernias in pregnant patients has yet to be established, but it is clear that each case must be dealt with on an individual basis. It is essential for surgeons to consider the length of the surgery, the presence of contamination, the age of the fetus, the size of the defect, and the chances of recurrence before selecting which technique to employ when repairing abdominal wall hernias in pregnancy.
\end{abstract}

Keywords: Hernia in pregnancy; Strangulated recurrent incisional hernia; Strangulated incisional hernia; Recurrent incisional hernia; Incisional hernia

\section{Introduction}

Incisional hernias appear at a rate of $18.7 \%$ by the 10 -year follow-up [1]. However, the incidence of these hernias during pregnancy is unknown as only case reports exist in the literature [1]. In a pregnant patient, the clinical presentation

\footnotetext{
Manuscript accepted for publication October 2, 2013

${ }^{\text {a}}$ Trauma Services, St. Elizabeth Health Center, Youngstown, OH, USA

${ }^{\mathrm{b}}$ Trauma and Orthopaedic Trauma Research, St. Elizabeth Health Center, Youngstown, OH, USA

${ }^{\mathrm{c}}$ Corresponding author: Heath A. Dorion, St. Elizabeth Health Center, 1044 Belmont Avenue, Youngstown, OH 44501, USA.

Email: Heath_Dorion@hmis.org
}

doi: http://dx.doi.org/10.4021/jmc1504w of a hernia is not always clear cut. One may confuse the often variable and vague symptoms with morning sickness or uterine contractions $[2,3]$. It is critical that physicians thoroughly examine this patient population for the possibility of a hernia to avoid potentially adverse and even morbid outcomes for the mother and fetus. Additionally, if a pregnant patient presents with a known history of gastric bypass surgery, then the physician should be highly suspicious of a hernia [2]. The use of computed tomography (CT) to investigate the source of the symptoms is acceptable, as it produces minimal risk to the fetus $[2,4]$. Even if the $\mathrm{CT}$ is negative but there is still reason to suggest a hernia, then exploratory laparotomy should be discussed with the patient $[2,4]$. A delay in the surgery could result in morbid outcomes [5].

Managing these hernias depends on the emergent nature of the situation and the gestational age at the time of presentation [1]. While hernias are often asymptomatic and can be treated conservatively during pregnancy, some may become incarcerated, strangulated, or irreducible requiring immediate surgical intervention $[1,6]$. The mortality rate is high for both the mother and the fetus in the case of a strangulated hernia $[7,8]$. We report a case of a 26 -week gestation, morbidly obese patient who presented with a strangulated upper midline incisional hernia.

\section{Case Report}

A 33-year-old morbidly obese (body mass index $=42$ ), gravida 3 , para 1 , woman at 26 weeks gestation initially presented with a 24-h history of nausea, vomiting, and severe epigastric abdominal pain. Her surgical history included appendectomy, ovarian cystectomy, Caesarean section, open Roux-en-Y gastric bypass, and incisional hernia repair with absorbable mesh. Physical examination demonstrated a female in severe pain with a tender, large, non-reducible, epigastric mass immediately underneath her prior upper midline surgical scar. A CT scan demonstrated a strangulated upper midline incisional hernia (Fig. 1). A perinatology consultation confirmed no apparent fetal distress. The patient was then immediately taken to the operating room (OR) for exploration. A non-perforated, gangrenous, $25 \mathrm{~cm}$ segment of 


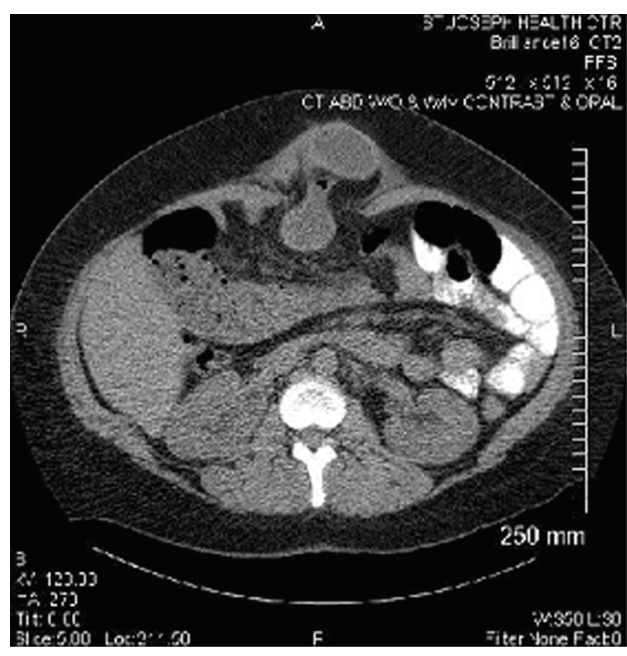

Figure 1. Initial CT scan shows an incarcerated midline hernia containing the small bowel.

ileum was discovered in the hernia sac. This segment was resected and a primary anastomosis was performed. The resulting fascial defect was approximately $7 \mathrm{~cm}$ wide and not amenable to primary suture repair. Therefore, an interposition $15 \mathrm{~cm}^{2}$ double layer absorbable mesh was implanted. Fortunately, she did not develop any pregnancy-related complications and had an elective Caesarean section at term.

Five months postpartum, she again developed a strangulated upper midline incisional hernia (third recurrence). She was taken to surgery where a non-perforated, gangrenous $30 \mathrm{~cm}$ segment of ileum was identified and resected. A $10 \mathrm{~cm}$ fascial defect was identified this time and repaired with a $120 \mathrm{~cm}^{2}$ underlay porcine small intestinal submucosa biologic implant. Her immediate postoperative course was uneventful.

During a clinic visit 8 months later, a reducible, recurrent upper midline hernia was detected (fourth recurrence). Laparoscopic incisional hernia repair was then attempted; however, the fascial defect appeared to be too large to adequately position an intraperitoneal mesh. Therefore, the procedure was converted to an open technique. A modified Rives-Stoppa repair with a $900 \mathrm{~cm}^{2}$ lightweight polypropylene mesh was performed - subcutaneous closed suction drains were inserted. The patient was discharged home 4 days later.

She was readmitted to the hospital on postoperative day (POD) 8 with a methicillin-resistant Staphylococcus aureus mesh infection (Fig. 2). Intravenous vancomycin was started, but the drain output remained purulent over the next 72 h. Therefore, she was taken to the OR on POD 11 for mesh removal and wound vacuum-assisted closure (VAC) application. The wound VAC was changed 4 days later, and the gross purulence in the wound had resolved. Subsequently, the wound VAC was removed, and her abdominal wall was repaired with a $300 \mathrm{~cm}^{2}$ non-cross-linked, cadaveric dermal

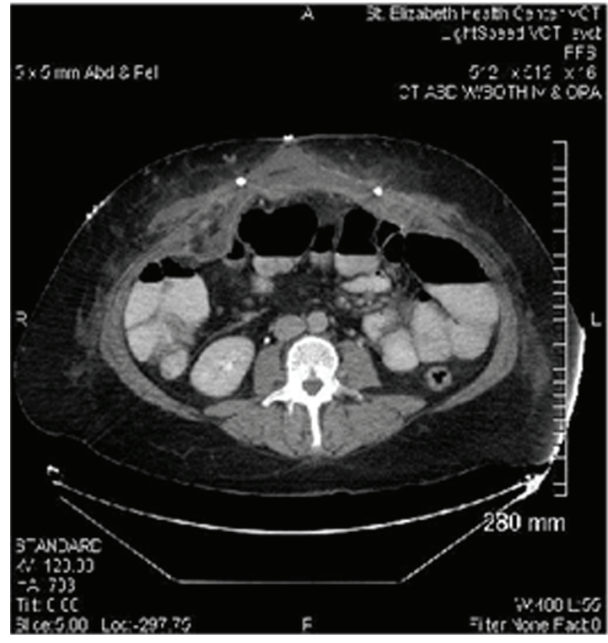

Figure 2. Readmission CT scan shows a fluid collection with surrounding inflammatory changes adjacent to the mesh, and surgical drains and skin staples are visible in the image.

underlay implant. The midline fascia was not reapproximated. The large skin flaps were tacked down with quilting sutures, and autologous platelet gel was applied to the flaps. She was discharged home 15 days later. The patient developed a postoperative seroma that required serial ultrasoundguided aspirations in the clinic over the course of 8 weeks.

Four months later, she presented to the clinic with her fifth recurrent incisional hernia and was taken to the OR for a component separation repair, which was reinforced with a non-cross-linked, porcine dermal biologic underlay implant. To date, she has no evidence of recurrent hernia but suffers from chronic abdominal and back pain.

\section{Discussion}

A strangulated incisional hernia in pregnancy is a rare event, and the incidence of uncomplicated incisional hernia in pregnancy has not been reported. Only one other similar case has been published. Young et al described a 34-week pregnant patient with bowel obstruction and incarceration that was successfully managed with a short course of preoperative betamethasone for its effect on fetal development, followed by simultaneous Caesarean section and primary suture repair of the hernia [1].

The ideal management of incisional hernia in pregnancy is unknown. Both open and laparoscopic hernia repair are possible in pregnancy. An open technique may be a more familiar and quicker approach while laparoscopic hernia repair may be more difficult in the presence of strangulated hernia. Since the Luijendijk et al study in 1990, the gold standard for incisional hernia repair in the general population is permanent, synthetic mesh reinforcement [2]. However, implanting synthetic mesh is relatively contraindicated in the set- 
ting of a contaminated surgical field. Primary suture repair is a safe option in pregnancy when the fascial defect can be closed without significant tension, although the recurrence rate is likely higher [2]. Hernia repair with an absorbable, synthetic mesh can also be used, particularly when a large defect is present, but again, recurrent hernia would be likely. Another consideration is to use a bioprosthetic mesh. These devices have been successfully used in contaminated and infected hernia operations [3]. However, the risk to the fetus when using a biologic implant for hernia repair is unknown. In addition, the cost of bioprosthetic mesh is high, and the effect of the increased biomechanical stress and strain of the gravid uterus on the abdominal wall-bioprosthetic interface is unpredictable.

The development of an infected hernia mesh is a devastating event for the patient. Risk factors include the following: patient age, American Society of Anesthesiologists score, tobacco use, and the duration and emergency setting of the operation [4]. The patient may present with pain, swelling, drainage, and fever during the immediate postoperative period, but the presentation may also be delayed for months to years after surgery, which is why the diagnosis is typically based on clinical findings. CT scanning is sometimes helpful to distinguish between recurrent hernia and an infected appearing fluid collection around the mesh, but a culture of the fluid is diagnostic. Offending organisms are usually staphylococcus, enterococcus, and Gram-negative bacteria. The presence of enteric flora should raise the suspicion of a bowel-mesh fistula. While antibiotics alone may be attempted to treat the infection, definitive management usually necessitates partial or complete mesh removal and intravenous antibiotics. Subsequently, the resulting fascial defect may be quite extensive. Abdominal wall component separation has been used successfully in these situations for definitive repair [5]. One must consider each case individually and account for the length of the operation, the presence of contamination, the gestational age, the size of the defect, and the likelihood of recurrence when deciding on the technique to repair abdominal wall hernias in pregnancy.

\section{Acknowledgement}

The authors would like to thank Marina C. Hanes, B.A. and
Elisha A. Chance, B.S.A.S. for their assistance with this manuscript.

\section{Financial Support and Conflict of Interests}

No financial support was received for this study, and there are no conflicts of interest.

\section{References}

1. Young BC, Fugelso D, Takoudes T. Incisional hernia with bowel incarceration and obstruction at 34 weeks gestational age. Arch Gynecol Obstet. 2009;279(6):905907.

2. Luijendijk RW, Hop WC, van den Tol MP, de Lange DC, Braaksma MM, JN IJ, Boelhouwer RU, et al. A comparison of suture repair with mesh repair for incisional hernia. N Engl J Med. 2000;343(6):392-398.

3. Franklin ME, Jr., Trevino JM, Portillo G, Vela I, Glass JL, Gonzalez JJ. The use of porcine small intestinal submucosa as a prosthetic material for laparoscopic hernia repair in infected and potentially contaminated fields: long-term follow-up. Surg Endosc. 2008;22(9):19411946.

4. Mavros MN, Athanasiou S, Alexiou VG, Mitsikostas PK, Peppas G, Falagas ME. Risk factors for mesh-related infections after hernia repair surgery: a meta-analysis of cohort studies. World J Surg. 2011;35(11):2389-2398.

5. Collage RD, Rosengart MR. Abdominal wall infections with in situ mesh. Surg Infect (Larchmt). 2010;11(3):311318.

6. Torres-Villalobos GM, Kellogg TA, Leslie DB, Antanavicius G, Andrade RS, Slusarek B, Prosen TL, et al. Small bowel obstruction and internal hernias during pregnancy after gastric bypass surgery. Obes Surg. 2009;19(7):944-950.

7. Moore KA, Ouyang DW, Whang EE. Maternal and fetal deaths after gastric bypass surgery for morbid obesity. $\mathrm{N}$ Engl J Med. 2004;351(7):721-722.

8. Wai PY, Ruby JA, Davis KA, Roberts AC, Roberts KE. Laparoscopic ventral hernia repair during pregnancy. Hernia. 2009;13(5):559-563. 